\title{
Un estudio exploratorio sobre la donación hipotética de embriones humanos en Chile
}

\author{
J. A. ÁLVAREZ DÍAZ \\ Departamento de Historia de la Medicina. Universidad Complutense. Madrid
}

\section{RESUMEN}

Objetivo: Explorar opiniones de pacientes que han acudido a TRA complejas, respecto a la donación de gametos y embriones, así como las razones para hacerlo o no.

Pacientes y métodos: La sede fue el Hospital Clínico de la Universidad de Chile. Se entrevistaron diez participantes (siete mujeres, tres hombres), quienes habían acudido al menos a una TRA, sin formar parte de programas de donación. Se trata de un estudio transversal de bioética descriptiva, realizado con metodología cualitativa etnográfica mediante entrevista semiestructurada aplicando análisis del discurso al texto resultante.

Resultados: Respecto a la donación de embriones, seis participantes aceptarían donarlos, cinco con fines de terapia de fertilidad y uno con fines de investigación. En relación con criopreservación, tres participantes la aceptarían siempre, y tres con restricciones, de los cuales uno preferiría descartar en lugar de donar un embrión criopreservado.

Discusión: Puede sugerirse que: la donación de gametos es más comentada y generalmente aceptada; la donación de embriones es un tema menos discutido y más conflictivo al momento de pensar acerca de él, tanto para donar como para aceptar; la criopreservación es un tema complejo, comentado pero también conflictivo, cuya aceptación o no, así como el destino de los embriones probablemente criopreservados, depende de las concepciones que se tienen respecto al origen de la vida, la ética personal, etc.; se puede plantear que una hipótesis construida, a partir de este estudio y por verificarse en estudios cuantitativos, es que la donación de embriones podría efectuarse, mayormente para terapia de fertilidad, y excepcionalmente con fines de investigación.

PALABRAS CLAVE: Disposición de embriones. Técnicas de reproducción asistida (TRA). Fecundación in vitro (FIV). Inyección intracitoplásmica del espermatozoide (ICSI). Criopreservación. Chile.

\author{
AN EXPLORATORY STUDY REGARDING THE HYPOTHETICAL \\ HUMAN EMBRYO DONATION IN CHILE
}

\begin{abstract}
Objective: To explore opinions of patients who undergone to complex ART towards gamete and embryo donation, as well as the reasons to do it or not.

Patients and methods: The seat was the Hospital Clínico de la Universidad de Chile. There were interviewed ten participants (seven women, three men), who had undergone at least to one ART, without comprising of donation programs. It was a cross-sectional study of descriptive bioethics, done with ethnographic qualitative methodology with a semistructured interview applying speech analysis to the resulting text.

Results: Regarding embryo donation, six participants would accept to donate them, five to fertility therapy and one to research. Regarding the cryopreservation, three participants would always accept it, and three with some restrictions, just one on them would rather to discard instead of donating a cryopreserved embryo.

Discussion: It could be suggested: gamete donation is more commented and generally accepted; embryo donation is a more conflicting and less discussed subject, as much to donate as to accept; cryopreservation is a complex subject, commented but also conflicting, whose acceptance or not, as well as the destiny of the probably cryopreserved embryos, depends on the believes that participants have about the origin of the life, personal ethics, and the religion. It could be possible to say that a hypothesis constructed in this study (to be verified in future quantitative researches) is that embryo donation could take place, for therapy of fertility, and exceptionally to research.
\end{abstract}

KEY WORDS: Embryo disposition. Assisted reproductive technologies $(A R T)$. In vitro fertilization (IVF). Intracytoplasmic sperm injection (ICSI). Cryopreservation. Chile.

Álvarez Díaz JA. Un estudio exploratorio sobre la donación hipotética de embriones humanos en Chile. An Med Interna (Madrid) 2007; 24: 579-584.

\section{INTRODUCCIÓN}

Esterilidad (primaria o secundaria) es la incapacidad del varón o de la mujer de fecundar (1). Infertilidad (primaria o secundaria) es la incapacidad para parir un hijo vivo. Como el término "infertilidad" habla de "incapacidad", se utiliza cada vez más el término de subfertilidad. En Chile, luego de 8 años de matrimonio, aproxi- madamente una de cada 25 mujeres es subfértil (2). Este grupo de la población se ha beneficiado con el tratamiento con técnicas de reproducción asistida (TRA) simples (inseminación intrauterina, ya sea con semen del esposo o de donador) y complejas (fecundación in vitro con transferencia de embriones -FIV- e inyección intracitoplásmica del esperma al citoplasma del ovocito -ICSI-, así como variantes de estas técnicas). 
La FIV fue desarrollada por Steptoe y Edwards en Inglaterra, culminando con el nacimiento de Louise Joy Brown en 1978 (3). Chile publica el primer nacimiento de un producto vivo conseguido mediante FIV en 1984 (4). Ante esta nueva realidad terapéutica, se hacen recomendaciones desde el Ministerio de Salud respecto a la aplicación de la técnica, que incluían revisiones por parte de comités de ética (5). Tempranamente se empiezan las reflexiones respecto a la eticidad de la transferencia y la criopreservación, desarrollándose la transferencia en estado de pronúcleos (6), así como la de cigotos (7). Con el tiempo, comienzan a aparecer los primeros análisis del desarrollo de la experiencia chilena, así como lo que se podría esperar de la aplicación de estas TRA (8). Respecto a la micromanipulación de gametos, la ICSI es reportada en Italia hasta 1992 (9).

Por otro lado, en 1983 Trounson consigue en Australia el primer nacimiento resultado del embarazo con un embrión criopreservado (10). Esto cambia las TRA, ya que así existía la posibilidad de que la mujer no se sometiera a todo el tratamiento completo: bastaba que se presentara a la transferencia embrionaria.

La criopreservación de embriones suponía un ahorro económico y de estrés (físico y psicológico) a la mujer al evitar la hiperestimulación ovárica, ahorro en los medios de cultivo para que se realizara la FIV; se evita estrés al varón en la recolección de la muestra, etc. La criobiología ha tenido un desarrollo importante, existiendo la posibilidad de criopreservar espermatozoides, ovocitos, células en estado de pronúcleos, embriones desde 2 células hasta blastocistos, tejido ovárico y testicular. Esto ha hecho el reflexionar sobre la ética en la aplicación de la criobiología (11). Además, el contar con la posibilidad de mantener un embrión criopreservado genera un replanteamiento de muchos problemas importantes: filosóficos y teológicos, tales como la consideración sobre el momento de la animación (o infusión del alma espiritual al cuerpo) (12), y bioéticos, como la posibilidad de poder realizar investigación en embriones humanos (13). Asumiendo ciertos criterios éticos, Chile ha optado por la criopreservación de células en estado de pronúcleos (14).

Actualmente la donación de embriones es una realidad en países desarrollados. Las sociedades que agrupan profesionales de la medicina reproductiva han propuesto guías y recomendaciones éticas básicas a los centros que llevan a cabo los procedimientos, tanto en USA a través de la American Society for Reproductive Medicine (15), como en Europa a través de la European Society for Human Reproduction and Embryology (16).

A partir de la nueva realidad de combinar FIV con criopreservación, aparece un nuevo problema: ¿qué hacer con los embriones que están criopreservados? Las posibilidades se resumen en tres: que sigan criopreservados hasta que las parejas decidan utilizarlos para sí mismas con fines de reproductivos, que decidan donarlos (con fines reproductivos, de investigación o terapéuticos) o que decidan descartarlos. De esta manera es que se han iniciado investigaciones ante la realidad que presentan las naciones desarrolladas: un número considerable de individuos y parejas con embriones criopreservados.

La realidad latinoamericana es distinta por muchos factores: económicos, culturales, religiosos, sociales, etc. (17). Históricamente, las TRA y sus avances llegan en fechas posteriores a Latinoamérica. Económicamente no es accesible a toda pareja el costear un ciclo para una TRA, o incluir la criopreservación de embriones. Socialmente es muy difícil que una mujer sola acuda a una TRA. Los programas de donación son un tanto distintos (prácticamente no hay programas de donación de embriones). Respecto a la vivencia de la religiosidad, el cristianismo es predominante (de éste la forma mas común es el catolicismo, generalmente más del $80 \%$ de la población total) y su influencia tiene aún un peso importante en algunas de las decisiones personales de muchas parejas (de los participantes entrevistados se puede comentar que hay una cierta adecuación de su religiosidad, ya que la participación en una TRA muestra que no siguen al pie de la letra las enseñanzas del Magisterio de la Iglesia, pero no puede negarse que su influencia puede estar presente en otras áreas de su vida personal, como consideraciones respecto al embrión, etc.). Por otro lado, la situación es similar en cuanto a que muchos países cuentan ya con centros que realizan TRA y que incluyen dentro de los servicios la criopreservación.

En Latinoamérica el problema de los embriones criopreservados no es cuantitativamente tan severo como en las naciones desarrolladas. Se ha propuesto que, de no considerar esta reflexión tanto desde el punto de vista bioético como biojurídico, Latinoamérica podría convertirse en un mercado para naciones desarrolladas: de venta de gametos y de compra de embriones. La legislación que aborde el tema específico de la donación de embriones humanos es prácticamente nula en Latinoamérica.

Lo expuesto hasta el momento explica, al menos en parte, que no exista a la fecha ningún trabajo que remita a saber si las parejas latinoamericanas donarían sus embriones. Por ello, el objetivo del presente trabajo es realizar un estudio piloto para explorar opiniones de pacientes que han acudido a TRA complejas (FIV e ICSI) en Chile, respecto a la donación de embriones, así como las razones para hacerlo o no, con la idea de generar hipótesis para estudios posteriores de corte cuantitativo.

\section{PACIENTES Y MÉTODO}

El proyecto se desarrolló en el Hospital Clínico de la Universidad de Chile "José Joaquín Aguirre", Servicio de Obstetricia y Ginecología, Programa de Fertilización Asistida. Se contó con la aprobación del Comité de Ética, respetando normativa nacional chilena e internacional (Helsinki y CIOMS).

El Programa de Fertilización Asistida se inicia en 2003, y para su primer año ya había atendido a un universo de casi 100 pacientes, aproximadamente la mitad de fuera del área metropolitana de Santiago. La muestra seleccionada fue seleccionada en 2004 (al año de haberse iniciado el Programa) y de tipo no probabilística. Los criterios de inclusión fueron que se tratara de pacientes: cuyo su diagnóstico de subfertilidad les hubiera hecho tributarias de tratamiento con TRA complejas (no se consideraron TRA simples, como el caso de las inseminaciones), haber acudido al menos a un ciclo iniciado de tratamiento, y acudir al hospital para responder una entrevista. Los criterios de exclusión fueron que hubieran participado en un programa de donación.

En una primera fase para el proceso del consentimiento informado, el Servicio de Obstetricia y Ginecología, a través del Programa de Fertilización Asistida, envió una carta a todos los pacientes que habían participado en una TRA como sensibilización y primera invitación. Posteriormente se realizó una llamada telefónica, para reforzar la invitación y darles las 
generalidades de la investigación. En esta primera fase, la información era proporcionada por la matrona coordinadora del Programa.

En una segunda fase del proceso del consentimiento, los pacientes que aceptaron acudir al Hospital recibieron una explicación detallada por parte del investigador, y si aceptaban, firmaban un formato para evidenciar el proceso. Se ofrecía la opción de responder la entrevista en ese momento, o dar más tiempo en caso de requerirlo. En todos los casos los participantes respondieron tras la firma del formato de consentimiento informado. Una vez cubiertos los criterios, aceptaron participar ocho pacientes. Ante la baja respuesta, la matrona realizó una nueva invitación telefónica en la cual se solicitaba su autorización para que el investigador acudiera a su domicilio (para evitar su desplazamiento y hacerlo mas cómodo para ellos), aceptando solamente una pareja mas. De esta manera, finalmente se contó con diez pacientes, siete mujeres y tres hombres. En el caso de los varones, siempre se logró entrevistar a su pareja, no así en el de las mujeres.

La entrevista se realizó de forma individual a cada uno de los miembros de la pareja, se grabó en un medio electrónico, se transcribió textualmente, y al trascrito resultante se aplicó la metodología de análisis del discurso. En el análisis se buscaron intencionadamente los argumentos a favor o en contra de la donación.

\section{RESULTADOS}

Las características sociodemográficas y clínicas mas relevantes de los diez participantes se consignan en su género, edad, diagnóstico, la TRA a la cual acudieron, el número de intentos de dicha TRA, su nivel educativo, y la religión que referían practicar (Tabla I).

De acuerdo a la TRA a la cual se sometieron, cinco fueron a FIV y cinco a ICSI. De las cinco FIV, para tres fue su primer intento (uno sin implantación) y para dos fue su segundo intento (sin implantación). De los cinco ICSI, para dos fue su primer intento (sin implantación), y para tres fue su segundo intento (Tabla II). El rango de las edades fue de 26 a 41 años, con una media de 34.9 años, mediana de 34 , siendo una muestra bimodal (33 y 39 años).

Respecto a donar o recibir gametos donados las respuestas fueron muy consistentes. Tres participantes (dos mujeres y un hombre) no aceptarían nunca donar o recibir un gameto (óvulo o espermatozoide), mientras que los siete restantes (cinco muje-

\begin{tabular}{|c|c|c|c|c|}
\hline \multicolumn{5}{|c|}{ TABLA II } \\
\hline \multicolumn{5}{|c|}{$\begin{array}{c}\text { PARTICIPANTES DE ACUERDO A LA TRA, NÚMERO DE } \\
\text { CICLOS INICIADOS, RESULTADO, GÉNERO Y } \\
\text { EDAD (EN ARÁBIGO) }\end{array}$} \\
\hline \multicolumn{5}{|c|}{ Número de ciclo iniciado } \\
\hline & Primero & & Segundo & \\
\hline & Implantación & $\begin{array}{c}\text { Implantación } \\
+\end{array}$ & Implantación & $\begin{array}{c}\text { Implantación } \\
+\end{array}$ \\
\hline $\begin{array}{l}\text { Técnica de } \\
\text { fecundación }\end{array}$ & $\begin{array}{l}\text { FIV } \\
\text { ICSI }\end{array}$ & $\begin{array}{c}\text { F41 } \\
F 26, M 40\end{array}$ & $\mathrm{~F} 35, \mathrm{M} 32$ & $\begin{array}{l}\text { F31, F33 } \\
\text { F33, F39, } \\
\text { M39 }\end{array}$ \\
\hline
\end{tabular}

M: masculino; F: femenino

res y dos hombres) lo aceptarían siempre (Tabla III). Las personas entrevistadas fueron las mismas quienes aceptarían tanto la donación como la recepción hipotética de los gametos.

Para la donación de embriones las respuestas fueron un poco más variables. Si se trataba de aceptar un embrión donado como terapia para su problema de fertilidad, cuatro participantes (tres mujeres y un hombre) no aceptarían nunca, tres (dos mujeres y un hombre) aceptarían siempre un embrión donado como terapia para su problema de fertilidad, y los otros tres (dos mujeres y un hombre) aceptarían pero solamente bajo ciertas restricciones (como el anonimato, o aplicación de pruebas de tamizaje para descartar problemas genéticos hereditarios).

Cosa distinta fue en el momento de plantearse el donar los embriones propios: cuatro de los participantes (tres mujeres y un hombre) no aceptarían nunca donar un embrión, mientras que seis participantes aceptarían donarlos (cuatro mujeres y dos hombres), cinco (cuatro mujeres y un hombre) con fines de terapia de fertilidad y uno (hombre) con fines de investigación (con la limitación de que fuese para mejorar el éxito de las TRA).

Finalmente, en el tema de la criopreservación, cuatro participantes (tres mujeres y un hombre) no aceptarían nunca, tres participantes (dos mujeres y un hombre) la aceptarían siempre, y tres (dos mujeres y un hombre) con restricciones (mayormente que fuese con el fin de esperar a que en un ciclo posterior les fuesen transferidos a si mismos), de los cuales uno (mujer) preferiría descartar antes que donar un embrión criopreservado (Tabla IV).

TABLA I

PRINCIPALES CARACTERÍSTICAS SOCIODEMOGRÁFICAS Y CLÍNICAS DE LOS PARTICIPANTES

\begin{tabular}{|c|c|c|c|c|c|c|c|}
\hline Participante & Género & Edad & Diagnóstico sindromático & TRA & Ciclo iniciado & Educación & Religión \\
\hline 1 & $\mathrm{~F}$ & 39 & Endometriosis + Subfertilidad masculina & ICS & Segundo & Superior (fonoaudióloga) & Católica \\
\hline 2 & M & 39 & Endometriosis + Subfertilidad masculina & $|\mathrm{CS}|$ & Segundo & Superior (ingeniero) & Católica \\
\hline 3 & $\mathrm{~F}$ & 33 & Subfertilidad masculina & FIVTE & Segundo & Superior (educadora) & Católica \\
\hline 4 & $\mathrm{~F}$ & 41 & Factor ovárico & FIV & Primero & Superior (optometrista) & Ninguna \\
\hline 5 & $\mathrm{~F}$ & 33 & Subfertilidad masculina & $|\mathrm{CS}|$ & Segundo & Superior (administradora) & Católica \\
\hline 6 & $\mathrm{~F}$ & 33 & Factor tuboperitoneal & FIVTE & Segundo & Media (secretaria) & Católica \\
\hline 7 & $\mathrm{~F}$ & 26 & Subfertilidad masculina & $|C S|$ & Primero & Media (empleada) & Evangélica \\
\hline 8 & M & 40 & Subfertilidad masculina & $|C S|$ & Primero & Técnico (recepcionista) & Católico \\
\hline 9 & $\mathrm{~F}$ & 34 & Factor tuboperitoneal & FIVTE & Primero & Superior (turismo) & Ninguna \\
\hline 10 & M & 32 & Factor tuboperitoneal & FIVTE & Primero & Superior (administrador) & Católico \\
\hline
\end{tabular}


TABLA III

PARTICIPANTES DE ACUERDO A LA ACEPTACIÓN EN LA DONACIÓN Y RECEPCIÓN DE GAMETOS

\begin{tabular}{|c|c|c|c|c|c|c|c|c|}
\hline \multicolumn{5}{|c|}{ Donación de gametos } & \multicolumn{4}{|c|}{ Recepción de gametos } \\
\hline & Espermatozoides & & Óvulos & & Espermatozoides & & Óvulos & \\
\hline & Aceptación & Rechazo & Aceptación & Rechazo & Aceptación & Rechazo & Aceptación & Rechazo \\
\hline Masculinos & 2 & 1 & 2 & 1 & 2 & 1 & 2 & 1 \\
\hline Femeninos & 5 & 2 & 5 & 2 & 5 & 2 & 5 & 2 \\
\hline
\end{tabular}

TABLA IV

PARTICIPANTES DE ACUERDO A LA ACEPTACIÓN EN LA DONACIÓN Y RECEPCIÓN DE EMBRIONES

\begin{tabular}{lcccccc}
\hline & \multicolumn{3}{c}{ Donación de embriones } & \multicolumn{2}{c}{ Recepción de embriones } \\
\hline & $\begin{array}{c}\text { Terapia de } \\
\text { fertilidad }\end{array}$ & Investigación & Rechazo & Aceptación & $\begin{array}{c}\text { Aceptación con } \\
\text { restricciones }\end{array}$ & Rechazo \\
\hline Masculinos & 1 & 1 & 1 & 1 & 1 & 1 \\
Femeninos & 4 & 0 & 3 & 2 & 2 & 3 \\
\hline
\end{tabular}

\section{DISCUSION}

Los datos disponibles en la literatura se refieren a estudios realizados principalmente en países desarrollados y en pacientes que tienen embriones criopreservados, pero son con los que se cuenta hasta el momento para citar y comparar con estos datos nuevos. Un estudio realizado en USA con 365 pacientes, el $43 \%$ preferiría descartarlos, $11 \%$ donaría a otras parejas, y un $10 \%$ donaría con fines de investigación (18). En Australia se pueden citar varios estudios. En uno de ellos, realizado con 1246 parejas, el $89.5 \%$ preferiría descartar sus embriones (19); otro, realizado con 509 parejas, mostraba que el $34 \%$ preferiría donar sus embriones para investigación médica (20); otro estudio con 126 pacientes muestra que un $30 \%$ donaría sus embriones para realizar investigación (21); uno mas revisa el estado del arte y consigna que el $90 \%$ preferiría descartar en lugar de donar sus embriones (22); finalmente, otro estudio realizado con 311 parejas concluye que el $42 \%$ donaría sus embriones con fines de investigación (23). En Italia, una revisión de la experiencia en 10 años muestra que solamente el $6 \%$ de los pacientes donaría sus embriones (24). En Dinamarca, un estudio realizado en 207 parejas mostró que (resultados que no son mutuamente excluyentes) un $28,5 \%$ donaría sus embriones a una pareja infértil, 60,4\% para investigación en infertilidad, 56,5\% para investigación en células troncales, y 48,8\% para tratamiento con células troncales (25). Con estos datos se puede notar algo en común: en naciones desarrolladas parece ser más sencillo descartar los embriones supernumerarios, o en su caso, donarlos con fines de investigación, que donarlos para terapia de fertilidad. Esto llama la atención porque en definitiva no coincide con lo encontrado en este estudio cualitativo en una nación menos desarrollada. Ahora se confronta esto un poco más a detalle.

Aunque no es frecuente la utilización de metodología cualitativa, también el campo de la medicina reproductiva ha notado la deficiencia de este tipo de estudios, por lo que han aparecido cada vez más con este enfoque, explorando temas como la vivencia de la subfertilidad (26) o de las TRA (27). Al realizar estudios cualitativos respecto a la donación de embriones, en USA se ha hecho notar que las actitudes del tipo de participantes que se incluyen en esta investigación no es un tema explorado (28). Otro estudio cualitativo realizado en USA se refiere a las conceptualizaciones que tienen los padres respecto a los embriones (29), las cuales varían desde considerar a los embriones criopreservados como tejido biológico, entidades vivientes, "niños virtuales" con intereses que deben ser considerados y protegidos, hermanos de los hijos nacidos, hasta recuerdos simbólicos de su subfertilidad pasada. Para este estudio ninguno de los participantes consideró a sus embriones como tejido biológico, ni como recuerdo de su subfertilidad. Las opiniones respecto a considerar los embriones como tejido biológico fueron escasas, tales como "se unieron espermatozoide óvulo y ya hay una, una división celular". La mayoría consideró que los embriones tenían más relación con un ser humano equiparable a un hijo, con expresiones tales como "dejar embriones ahí que yo no sé si voy a poder recuperar después, es como dejar a los hijos ahí abandonados, a la deriva", "un embrión ya estamos hablando de un hijo con mi esposo", "yo creo que son como mis hijitos, es más, los que tengo yo ahora, los tengo hace mucho tiempo abandonados", etc. Quien prefería descartarlos en lugar de donarlos comentó "prefiero descongelarlos y que no sean ocupados, pero no donarlos", en una argumentación que iba también en el sentido de equiparar al embrión con un hijo nacido: es preferible saberlo muerto que no saber de él en absoluto. Quien opinó a favor de la donación de embriones con fines de investigación comentó que "yo creo que uno no puede ser egoísta, y si se pueden ganar oportunidades de tener una familia con experimentación, yo creo que sí, es tanto como la opción de donar embriones a parejas como para estudio. Y si fueran como demasiados los embriones, yo lo haría". Esto coincide con observaciones en otros estudios respecto a pensamientos altruistas que se dirigen primordialmente a parejas en una situación similar a la que han padecido este grupo estudiado: la vivencia de la subfertilidad en distintas maneras.

Aunado a esto, también resulta interesante que los profesionales de la medicina reproductiva en Latinoamérica parecieran coincidir en parte con las afirmaciones de los pacientes entrevistados. La Red Latinoamericana de Reproducción Asistida (red LARA) se reunió en 1995 en Reñaca, Chile, para 
trabajar sobre un "Consenso latinoamericano en aspectos ético-legales relativos a las técnicas de reproducción asistida", publicado al año siguiente. En este documento se indica que "La comunidad científica latinoamericana no intentó definir los inicios biológicos de una persona, de hecho, no es a través de la biología que se llega a esta definición. A pesar de ello, existe consenso que el camino a ser persona se inicia una vez completada la fertilización y establecida la individualidad genética" (30). Por este motivo es que varios hospitales adheridos a la red LARA criopreservan células en estado de pronúcleos, al considerar que aun no se encuentran ante ese estado de "camino a ser persona" por no haber ocurrido la primera división mitótica. Además, el documento señala que "Todo programa de criopreservación debe ir ligado a un programa de donación o adopción de concepti que permita encontrar una madre para aquellos concepti que no serán transferidos a sus progenitoras". De esta manera es entendible que la red haya generado modelos de consentimiento informado donde se considere esta situación. En el texto propuesto para la "criopreservación de células en estado de pronúcleo" dice "En caso de decidir no hacer uso de estas células en estado de pronúcleos o embriones, en un plazo de 3 años, desde la criopreservación y habiéndolo manifestado por escrito, la pareja debe autorizar a la Unidad de Medicina Reproductiva, para donarlas en forma confidencial y anónima a parejas infértiles que deseen recibirlas en adopción. Las células en estado de pronúcleo no serán destruidas" (31). Estas observaciones resultan importantes, ya que durante la entrevista no parecía que estos textos hubiesen influenciado las opiniones de los participantes, sino que más bien parecía que coincidían con ellos, al menos en parte. Además, la distinción que la red hace entre células en estado de pronúcleos y el embrión no estaba presente en ninguno de los entrevistados.

El primer estudio que intenta una aproximación al tema de la donación de embriones fue realizado también en Chile, donde se exploró la ansiedad que despierta la idea de la donación hipotética de embriones con fines de investigación, encontrándose que los tipos de ansiedad más frecuentemente asociados fueron la ansiedad de culpa, de separación y de mutilación (32). Esto muestra que el proceso psicológico de enfrentar esta situación es complicado; en parte, por creencias donde se "personaliza" al embrión. Posteriormente un estudio similar encontró que los tipos de ansiedad más comunes en población peruana, fueron la ansiedad de culpa, la ansiedad de separación y la ansiedad de vergüenza (33). Con un análisis desde la perspectiva de género, se sugirió que algunas diferencias en la ansiedad generada dependían de factores asociados a la construcción de las masculinidades y las feminidades en los participantes entrevistados (34). Estos estudios, junto a reflexiones teóricas, llevaron a proponer la necesidad de generar políticas públicas explícitas en relación con la investigación con embriones humanos (35), terreno aun menos explorado en Latinoamérica.

Finalmente, del grupo estudiado se puede comentar que la donación de gametos es un tema más comentado y generalmente aceptado por los participantes; la donación de embriones es un tema menos discutido y más conflictivo al momento de pensar acerca de él, para recibirlos y aún más para donarlos; la criopreservación es un tema complejo, comentado pero también muy conflictivo, cuya aceptación o no, así como el destino de los embriones probablemente criopreservados, depende de las concepciones que tienen los participantes respecto al origen de la vida, su ética personal, su construcción del género y su forma personal de vivir su religión (ya que las opiniones personales vertidas en las entrevistas no necesariamente reflejan las posturas oficiales de la religión con la cual se identificaban o decían practicar). Con todo lo anterior, se puede plantear que las hipótesis que pueden construirse desde este estudio cualitativo para poder ser contrastadas en un futuro con una muestra estadísticamente representativa de esta población y poder sugerir generalizaciones, serían que la donación de embriones podría efectuarse, principalmente con fines de terapia de fertilidad, y excepcionalmente con fines de investigación. La importancia de verificar esto en el futuro es, como se hiciera notar previamente, que en la mayoría de estudios disponibles realizados en países desarrollados la donación con fines de terapia de fertilidad es minoritaria, mientras que descartar los embriones o donarlos para investigación y/o experimentación es predominante.

\section{Bibliografía}

1. Fathalla MF, Rosenfield A, Indriso C, Sen DK, Ratman SS. Salud reproductiva: Problemas mundiales. En: Rosenfield A, Fathalla F (ed). Manual de Reproducción Humana (FIGO). Madrid: The Partenon Publishing Group; 1994. p. 386-99.

2. Fuentes A, Devoto L. Infertility after 8 years of marriage: a pilot study. Hum Reprod 1994; 9: 273-8.

3. Steptoe PC, Edwards RG. Birth after implantation of a human embryo. Lancet 1978; 2: 366.

4. Costoya AA, Schmitt YJM, Rey GM, Dujoune CS, Sánchez UMI, Godan MA, et al. Embarazo obtenido por fertilización "in vitro" y transferencia embrionaria. Rev Chil Obstet Ginecol 1984; 49: 206-16.

5. Chile. Ministerio de Salud. Ministerial Directive No. 1072, June 1985. Annu Rev Popul Law 1988; 15: 53.

6. Crisosto C, Cheviakoff S, Vera JA, Rutllant J, Arguello B, Romero C, Barros C. (In vitro fertilization program. Preliminary results) Rev Chil Obstet Ginecol 1989; 54: 375-80.

7. Vera JA, Crisosto C, Rutllant J, Barros C, Cheviakoff S, Arguello B. [The results of a program of in vitro fertilization and tubal embryo transfer (ZIFT)] Rev Chil Obstet Ginecol 1992; 57: 247-52.

8. Cheviakoff S, Vera JA. (What results can be expected from in vitro fertilization and other technics of assisted fertilization?) Rev Chil Obstet

Ginecol 1992; 57: 362-9.

9. Palermo G, Joris H, Devroey P, Van Steirteghem AC. Pregnancies after intracytoplasmic injection of single spermatozoon into an oocyte. Lancet 1992; 340: 17-8.

10. Trounson A, Mohr L. Human pregnancy following cryopreservation, thawing and transfer of an eight cell embryo. Nature 1983;305:707-709.

11. de Watcher MAM. Ethical aspects of cryobiology: Responsible applications in biomedicine and in clinical practice. Cryobiology 2004; 48: 205-13.

12. Cruz-Coke Madrid R. ¿Desde qué momento hay alma en el embrión humano? Rev Med Chile 2002; 130: 590-2.

13. Salinas PH, Adauy EA, Ide VR. Reflexiones acerca de células troncales obtenidas mediante la utilización de embriones humanos. Rev Chil Obstet Ginecol 2005; 70: 264-71.

14. Pommer TR, Arguello BB, Diaz-Fontevilla M, Martinez C, Fuentes GA, Furman I, et al. Criopreservación de pronúcleos: Rol en el programa de fertilización asistida. Rev Chil Obstet Ginecol 2003; 68: 287-92.

15. The Practice Committee of the American Society for Reproductive Medicine and the Practice Committee of the Society for Assisted Reproductive Technology. 2006 Guidelines for gamete and embryo donation. Fertil Steril 2006; 86: S38-S50. 
16. ESHRE Task Force on Ethics and Law. III. Gamete and embryo donation. Hum Reprod 2002; 17: 1407-8.

17. Zegers-Hochschild F. Attitudes towards reproduction in Latin America. Teachings from the use of modern reproductive technologies. Hum Reprod Update 1999; 5: 21-5.

18. Van Voorhis BJ, Grinstead DM, Sparks AE, Gerard JL, Weir RF. Establishment of a successful donor embryo program: medical, ethical, and policy issues. Fertil Steril 1999; 71: 604-8.

19. Kovacs GT, Breheny SA, Dear MJ. Embryo donation at an Australian university in-vitro fertilisation clinic: issues and outcomes. Med J Aust 2003; 178: 127-9.

20. McMahon CA, Gibson FL, Leslie GI, Saunders DM, Porter KA, Tennant CC. Embryo donation for medical research: Attitudes and concerns of potential donors. Hum Reprod 2003; 18: 871-7.

21. Burton PJ, Sanders K. Patient attitudes to donation of embryos for research in Western Australia. Med J Aust 2004; 180: 559-61.

22. Fuscaldo G, Savulescu J. Spare embryos: 3,000 reasons to rethink the significance of genetic relatedness. Reprod Biomed Online 2005; 10 : $164-8$.

23. Cattoli M, Borini A, Bonu MA. Fate of stored embryos: Our 10 years experience. Eur J Obstet Gynecol Reprod Biol 2004; 115: S16-S18.

24. Hammarberg K, Tinney L. Deciding the fate of supernumerary frozen embryos: a survey of couples' decisions and the factors influencing their choice. Fertil Steril 2006; 86: 86-91.

25. Bangsboll S, Pinborg A, Yding Andersen C, Nyboe Andersen A. Patients' attitudes towards donation of surplus cryopreserved embryos for treatment or research. Hum Reprod 2004; 19: 2415-9.

26. Dyer SJ, Abrahams N, Hoffman M, van der Spuy ZM. 'Men leave me as I cannot have children': Women's experiences with involuntary childlessness. Hum Reprod 2002; 17: 1663-8.

27. Arranz Lara L, Blum Grimberg B, Ito Sugiyama E. Estudio de un grupo de mujeres sujetas a tratamientos de reproducción asistida: Un enfoque cualitativo. Salud Ment 2001; 24: 30-6.

28. Lyerly A, Brelsford E, Bankowski B, Faden R, Wallach E. A qualitative study of individuals' attitudes regarding their cryopreserved embryos. Int Congr Series 2004; 1271: 353-6.

29. Nachtigall RD, Becker G, Friese C, Butler A, MacDougall K. Parents' conceptualization of their frozen embryos complicates the disposition decision. Fertil Steril 2005; 84: 431-4.

30. Red Latinoamericana de Reproducción Asistida. Consenso latinoamericano en aspectos ético-legales relativos a las técnicas de reproducción asistida. Cad Saúde Públ Rio de Janeiro 1998; 14: 140-6.

31. Red Latinoamericana de Reproducción Asistida. Formularios de educación y consentimiento en procedimientos de reproducción asistida. 2001. Disponible en URL: http://www.redlara.com/fdash45o9.asp?arq=Consentimientos\%20informados.PDF

32. Álvarez-Díaz JA. Tipos de ansiedad de las parejas tratadas con fertilización asistida frente a la donación hipotética de embriones en el Hospital Clínico de la Universidad de Chile. Ginecol Obstet Mex 2005; 73: 163 72.

33. Álvarez-Díaz JA. Tipos de ansiedad de las parejas tratadas con fertilización asistida frente a la donación hipotética de embriones en una clínica privada en Lima, Perú. Gac Med Mex 2006; 142: 223-8.

34. Álvarez-Díaz JA. Tipos de ansiedad frente a la donación hipotética de embriones para investigación. Una aproximación desde la perspectiva de género. Salud Ment 2006; 29: 59-65.

35. Álvarez-Díaz JA. Políticas públicas e investigación en embriones humanos. Rev Fac Med (Mex) 2005; 48: 242-7. 